\title{
Option Replication and the Performance of a Market Timer*
}

\author{
Georges HÜBNER ${ }^{\dagger}$
}

May 2015

\begin{abstract}
The Treynor and Mazuy framework is a widely used return-based model of market timing. However, existing corrections to the regression intercept can be manipulated through derivatives trading. We propose an adjustment based on Merton's option replication approach. The linear and quadratic coefficients of the regression are exploited to assess the cost of the replicating option that yields similar convexity for a passive portfolio. A similar reasoning applies for various timing patterns and in multi-factor models. The proposed framework induces a potential rebalancing risk and involves the delicate issue of choosing the cheapest option. We show that these issues can be overcome for reasonable tolerance levels.

Keywords: Performance measurement, market timing, Treynor and Mazuy, option replication, mutual fund performance.
\end{abstract}

JEL codes: G10, G12

${ }^{*}$ This paper has benefited from comments made by Hatem Ben Ameur, Maria Ceu Cortez, Georges GallaisHamonno, Marie Lambert, Bertrand Maillet, Guillaume Monarcha, Hery Razafitombo, as well as by participants in the Workshop on Investment Funds at the University of Luxembourg, the Thematic Cluster Day on Hedge Funds atf the University of Orleans, the 2011 French Finance Association Conference (Montpellier), and the 2011 European Financial Management Association Annual Meeting (Braga). I thank Thomas Lejeune and Arnaud Cavé for their excellent research assistance. Financial support from Deloitte Belgium and Deloitte Luxembourg is gratefully acknowledged. All remaining errors are my own.

${ }^{\dagger}$ Deloitte Chaired Professor of Portfolio Management and Performance, HEC Management School - University of Liège, Belgium; Associate Professor, School of Economics and Business, Maastricht University, the Netherlands; Founder \& Chief Scientific Officer, Gambit Financial Solutions Ltd, Belgium. Corresponding address. University of Liège, HEC Management School, Rue Louvrex 14 - N1, B-4000 Liège, Belgium. Phone: (+32) 42327428. E-mail: g.hubner@ulg.ac.be 


\section{Introduction}

Assessing the performance of actively managed funds is an ongoing theoretical and empirical challenge. According to the hypothesis of efficient financial markets, active fund managers would not be able to take advantage of proprietary skills that would generate reproducible positive abnormal returns. Traditional performance measures such as the Sharpe (1966) ratio, Jensen's (1968) alpha and the Treynor (1965) ratio reflect a clear aim to detect the manager's ability to perform superior asset selection. But when a fund's observed returns follow an unstable pattern, a fundamental question arises: is this mainly due to static exposures to non-linear instruments, or does it result from voluntary variations in the manager's betas, reflecting some kind of market timing skills? Unlike the majority of the hedge fund literature, studies on mutual fund performance have mostly taken the second route: viewing changing betas as an indication of market timing (see Jiang et al., 2007; Comer et al., 2009; Elton et al., 2012; Rodriguez, 2014) ${ }^{1}$, which is considered to be a portfolio management skill. The competing explanation, which attributes risk shifting to the use of derivatives as a way to create non-linear risk exposure, does not a priori correspond to superior performance. By trading option-like securities, the manager can mechanically create convexity or concavity in the portfolio returns. If portfolio performance is measured using traditional methods, such as Jensen's alpha or the Sharpe ratio, the output might give the illusion of superior skills when in reality the manager has "only" manipulated returns. Consider, for instance, a portfolio manager benchmarked on the S\&P500 index, who would systematically go long on the index for $101 \%$ of its equity, short $1 \%$ of ATM index calls with a maturity of 2 weeks, and would leverage the portfolio to get a beta of 1. Rebalancing this strategy - which involves no particular skill - from 1999 to June 2014 would deliver a statistically significant Jensen's alpha (measured with the market model) of $2.89 \%$ per year. The portfolio Sharpe ratio would reach 0.43 per year, in comparison with the index Sharpe

\footnotetext{
${ }^{1}$ In the context of hedge fund returns, which are usually reported with monthly frequencies, the vast majority of the literature refers to the first alternative (see Fung and Hsieh, 2001; Agarwal and Naik, 2004). Only a few recent papers (Chen and Liang, 2007; Detemple et al., 2010) explicitly associate hedge fund time-varying betas with a potential market timing behavior.
} 
ratio of 0.29 during the same period. The manager has not worked particularly well; she has only voluntarily or not - manipulated her portfolio performance.

To date, two return-based approaches have been used on a regular basis for the detection of market timing skills: the piecewise linear regression of Henriksson and Merton (1981) (henceforth HM) and the quadratic regression of Treynor and Mazuy (1966) (henceforth TM). ${ }^{2}$ Empirical evidence based on these measures has regularly delivered a disappointing picture regarding market timing skills (see Kryzanowski et al., 1996; Becker et al., 1999; Bollen and Busse, 2004; Comer et al., 2009). Furthermore, Krimm et al. (2011) find a negative relationship between timing and global performance. However, a direct holding-based approach delivers a more precise picture. Kaplan and Sensoy (2008) identify a positive relationship between variations in equity betas and market returns for market timing funds. In their study of detailed portfolio holdings, Jiang et al. (2007) conclude that the average performance attributable to timing skills is positive, and could amount to an annual level of $0.6 \%$. The extent of this abnormal return is economically relevant but the contrast of this result when set against the pervasive evidence is also empirically puzzling. Using a more granular analysis of holdings, Elton et al. (2012) conclude that most of the outperformance fades away when using a multi-index model. Even though these authors concede that market timing is also used by some fund managers, they find an average underperformance of market timers, mostly due to the managers with the most negative market timing behavior. A potential explanation could be provided by Huang et al. (2011), who show that risk-shifting of mutual fund portfolio holdings could be largely attributable to unskilled or manipulative managers. These managers tend to increase risk at the wrong time, leading to negative convexity in portfolio returns.

Reconciling the performance delivered by a simple model like the HM or TM with the one retrieved from a holding-based model is a more arduous task than merely comparing alphas. Ingersoll et al. (2007) demonstrate how easily one can manipulate most performance measures, including alphas, by dynamically trading securities to distort the distribution of returns. The resulting option-

\footnotetext{
${ }^{2}$ A noteworthy exception is the Positive Period Weighting Measure proposed by Grinblatt and Titman (1989), but this approach has led to few further developments in the literature on fund performance.
} 
like characteristics displayed by the pattern of portfolio returns has long been explicitly associated with spurious market timing effects (Jagannathan and Korajczyk, 1986). The mere separation between the regression intercept and the market timing coefficient in the HM and the TM models, as in Lee and Rahman (1990), is not sufficient to discriminate between genuine and false market timing skills. In order to better identify pure market timers, three types of adjustment of alphas have been proposed: a variance correction approach (Grinblatt and Titman, 1994); an approximation based on the squared benchmark returns (Bollen and Busse, 2004); and a synthetic option pricing approach (Merton, 1981). All three methods, however, remain subject to manipulation because a manager who has access to a complete derivatives market can easily alter the timing coefficient without affecting the regression intercept (alpha) to a proportional extent. Based on this statement, Ingersoll et al. (2007) propose a general formulation of a manipulation-proof performance measure (MPPM). Its functional form is contingent on the characterization of the investor's preferences.

In this paper, we revisit the Treynor and Mazuy model by applying, almost in its entirety, the original option replication approach proposed by Merton within the HM context. Our main objective is to derive the appropriate performance adjustment of the TM model, in the MPPM sense, in an economy where preferences are consistent with mean-variance analysis. Unlike all previous attempts to alter the TM regression alpha on the basis of the quadratic term alone, we exploit information on both the linear and the quadratic coefficients of the regression to assess the replicating cost of the option for a passively managed portfolio with non-linear benchmark exposures.

We use the same logic as in Ingersoll et al. (2007): if the manager has free access to a complete derivatives market on its benchmark, there are many ways in which she can distort the payoff of her portfolio. Considering the benchmark case where asset return distributions are stationary and Gaussian, the benchmark sensitivities of the option portfolio up to the second (quadratic) order can be approximated through a Taylor series expansion. Then, only the option delta, gamma and theta matter. Anyone can mix these option "greeks" to obtain the desired linear and quadratic sensitivities, but the same greeks will also entirely drive the periodic holding cost of this portfolio. 
Amongst all the portfolios involving derivatives that mimic a fund's pattern of returns, the cheapest one, called the "replicating portfolio" provides the minimum alpha required from a manager who claims to time the market. The actual performance of the active fund, mixing the impact of asset selection and market timing skills, is obtained by calculating the difference between its measured alpha and that of the replicating portfolio. In the simple case where only one option is used to replicate the market timing portfolio, we show that the performance measure has a simple and intuitive form. The approach can be easily extended to a multivariate setup without superfluous interaction terms, as in the extension of the TM model proposed by Lehmann and Modest (1987).

By setting the passive return as that of the cheapest option-based portfolio replicating strategy, this approach addresses the point of the identification of the option strategies raised by Ingersoll et al. (2007). Moreover, we overcome the performance manipulation issue through the use of options on traded securities. The availability of a complete market enables us to carry out an arbitrage argument, unlike in Ingersoll et al. (2007), whose proposed correction involves replicating a nontraded option on the quadratic market return.

The practical implementation of our approach involves tackling the delicate issue of choosing the appropriate replicating option characteristics. It involves a trade-off between the systematic risk and bias of the option portfolio rebalancing, on the one hand, and the minimization of the replication cost on the other. We posit that there are practical ways of dealing with this issue. Through an experiment on the U.S. market, the results suggest that the choice of an option maturing in 6 months' time provides a reasonable trade-off between rebalancing risk and replication cost when returns are measured on a weekly basis.

The paper is organized as follows. The second section introduces the existing approaches to account for market timing performance. In section 3, we develop the theoretical framework of portfolio replication with options from the Treynor and Mazuy model. Section 4 discusses the issues of option rebalancing and cost minimization. In section 5, we illustrate the choice of the replicating option. Section 6 concludes. 


\section{Performance measurement in market timing models}

The literature on one-factor models identifies two major specifications allowing the identification of a manager's market timing abilities. The Henriksson and Merton (1981) model considers that the manager chooses to switch the portfolio's beta depending on the sign of the market return. A good market timer increases the market exposure when the return is positive, and keeps it lower otherwise. Treynor and Mazuy (1966) propose the addition of a quadratic term to the one-factor model. The coefficient of this term reflects the convexity achieved by the manager through her exposure to the market portfolio. If this coefficient is positive, the manager gradually increases her beta as the market goes up, indicating that she is displaying a good timing ability. The regression equations of the Henriksson and Merton (1981) and the Treynor and Mazuy (1966) models are represented in equations (1) and (2), respectively:

$$
\begin{gathered}
r_{t}=\alpha_{\mathrm{HM}}+\beta_{\mathrm{HM}} r_{m t}+\gamma_{\mathrm{HM}}\left(-r_{m t}^{+}\right)+\varepsilon_{t} \\
r_{t}=\alpha_{\mathrm{TM}}+\beta_{\mathrm{TM}} r_{m t}+\gamma_{\mathrm{TM}} r_{m t}^{2}+\varepsilon_{t}
\end{gathered}
$$

where $r_{t} \equiv R_{t}-R_{f}$ is the portfolio excess return over the risk-free rate, $r_{m t}$ is the market portfolio excess return, and $-r_{m t}^{+}=\max \left(-r_{m t}, 0\right)$.

The HM model reflects the behavior of a manager who succeeds in switching her market beta from a high level equal to $\beta_{\mathrm{HM}}$ when the market return exceeds the risk-free rate to a low level of $\left(\beta_{\mathrm{HM}}-\gamma_{\mathrm{HM}}\right)$ otherwise. Admati et al. (1986) show that under the standard assumption of a joint normal distribution of asset returns, the TM model is consistent with a manager whose target beta varies linearly with her forecast for the expected market rate of return. In both models, a negative value of gamma induces negative market timing.

Both the HM and TM models aim to capture timing and selectivity skills using one single equation. The constant of the regression is supposed to capture the asset selection skills of the manager. The coefficient of the second variable (the truncated positive market return for HM and the squared market return for TM) reveals her ability to time the market, but this does not, in 
itself, deliver a measure of excess return due to market timing. To obtain a synthetic view on the manager's performance, it is necessary to perform an adjustment that simultaneously accounts for the timing and selectivity skills.

For the Henriksson and Merton (1981) model, an adjustment proposed by Merton (1981) perfectly corresponds to the exact cost of replicating the return generating process of equation (1). There exists no such perfect arbitrage-free formula for equation (2). Thus, in what follows, we will mostly discuss the performance measured in the Treynor and Mazuy (1966) model, which is the focus of the paper. To date, the literature on mutual fund performance has produced three alternative ways to measure the contribution of market timing to active returns: the first one based on the variance of market returns, the second one of the squared market returns (both approaches corresponding to ex-post adjustments), and the third one based on the value of an option.

\subsection{Ex-post mechanical adjustments to performance}

From the TM equation (2), two straightforward adjustments have been proposed in the literature. In summary, these adjustments result simply from estimating the mean of equation (2) and considering the fact that performance is obtained by calculating the difference between the average portfolio excess return and its linear required return.

The work of Admati et al. (1986) characterizes the properties of an active market timing portfolio managed optimally if returns are multivariate normal and the representative investor exhibits a constant absolute risk aversion (CARA) utility function. It is assumed that on receiving information about market returns, the manager linearly adjusts her beta to the timing signal. The authors show that the optimal portfolio returns are characterized by a quadratic function that can be measured by equation (2). In this particular instance of the TM model, the reward for the manager's market timing ability is appropriately represented by the variance of the market portfolio returns (Grinblatt and Titman, 1994), leading to equation (3) for the total return attributable to 
the manager's performance, denoted $\pi$ :

$$
\pi_{\mathrm{TM}, \mathrm{v}}=\alpha_{\mathrm{TM}}+\gamma_{\mathrm{TM}} \sigma_{m}^{2}
$$

where $\sigma_{m}^{2}$ is the variance of the market portfolio returns.

Within the framework proposed by Admati et al. (1986), the identification of the market timing premium is a step towards the identification of the coefficients of the regression, which corresponds to the optimal timing portfolio when the portfolio manager makes linear beta adjustments to timing signals (Grinblatt and Titman, 1994). This framework is thus restrictive. It entails an analytical connection between the regression coefficients $\alpha_{\mathrm{TM}}$ and $\beta_{\mathrm{TM}}$ in equation (2). Detemple et al. (2010) characterize this connection further by deriving a continuous-time generalization of market timing models, encompassing HM and TM as special cases. They derive a closed-form solution for the correlation structure between the returns of the fund and of the market, and they adapt equation (3) accordingly. The total performance adjusted for time-varying correlations collapses to expression (3) divided by the volatility of the portfolio returns.

An even simpler approach is proposed by Bollen and Busse (2004) and by Comer et al. (2009). These authors build upon the fact that a perfect market timer ex post would make a manager's beta time-varying according to market conditions by setting $\beta_{\mathrm{TM}, t}=\beta_{\mathrm{TM}}+\gamma_{\mathrm{TM}} r_{m, t}$. Accordingly, the total performance of a manager combining asset selection and market timing skills results from averaging the periodic market returns in the model with time-varying betas:

$$
\pi_{\mathrm{TM}, \mathrm{a}}=\alpha_{\mathrm{TM}}+\gamma_{\mathrm{TM}} \overline{r_{m}^{2}}
$$

Equation (4) is a simplified version of (3). The advantage of this formulation is that it is no longer restricted in order to correspond to an optimal behavior, as in the Admati et al. (1986) framework. However, the cost is a potentially high upward bias in the performance as measured with these metrics. When returns are computed with a daily frequency, the difference between the corresponding equations does not appear to matter empirically (Krimm et al., 2011). However, as the expected market return becomes significant with longer measurement intervals, the upward bias 
in equation (4) may start to matter.

Even though the formulations of the corrections are simple and seem to make sense, they appear to provide only a very crude adjustment to the portfolio alpha. Furthermore, this adjustment happens to be too small, which leads to an underestimation of true performance, because it rests on a flawed estimate of required return. To understand this, refer to equation (4). It corresponds to a performance equal to $\pi_{\mathrm{TM}, \mathrm{a}}=\bar{r}-\beta_{\mathrm{TM}} \overline{r_{m}}$, i.e. the mean portfolio return minus the required return under the linear market model. Because the fund generates a positive convexity (the quadratic term is always positive), it is less risky than suggested by its linear exposure only, and its required return must be lowered. $\pi_{\mathrm{TM}, \mathrm{a}}$ is thus too small. Note that, as $\overline{r_{m}^{2}}>\sigma_{m}^{2}$, the downward bias of equation (3) is even more pronounced. Naturally, if the manager generates a negative convexity, the outcome is opposite, and the fund's performance is overestimated.

To illustrate this misestimation of true market timing performance, we get back to the simple example developed in the first paragraph of the introduction. Calibrating a TM specification on the data would result in the following coefficient estimates: $\alpha_{\mathrm{TM}}=8.70 \%, \beta_{\mathrm{TM}}=0.984$ and $\gamma_{\mathrm{TM}}=$ -0.016 . Over the same period, we obtain the sample yearly values $\sigma_{m}^{2}=364.4$ and $\overline{r_{m}^{2}}=365.1$. The adjusted performance of the manager is thus estimated to be equal to $\pi_{\mathrm{TM}, \mathrm{v}}=3.04 \%$ and $\pi_{\mathrm{TM}, \mathrm{a}}=3.03 \%$, while in reality she displays absolutely no market timing. Those values barely differ from the alpha of $2.89 \%$ obtained with the linear market model. The mechanical adjustment is severely underestimated, leaving the impression of a good performance. The bias results from the inadequate assessments of the benefit of shorting the call options and the associated increase in risk due to the negative convexity of the fund's payoff.

In the next section, we present an alternative approach, which explicitly accounts for the role of the linear market beta in total performance with market timing. As for the original option-based approach proposed by Merton (1981), we associate the market timing return with the outcome of an option. Our analysis focuses on the cost, expressed in rates of return, of replicating the pattern of the TM regression using a mix of options and risk-free instruments only. 


\subsection{The replicating cost of building market timing portfolios}

Both the Admati et al. (1986) and the Bollen and Busse (2004) frameworks aim at directly measuring the contribution of market timing skills in the total portfolio return, irrespective of how the manager has tried to achieve this result. When considered from the point of view of the manager's intentions, her skill is related to her capacity to create an option on the market portfolio and to sustain this strategy over time.

The idea of calling upon option trading strategies to assess the market timing abilities of a portfolio manager is introduced by Merton (1981). In the context of the HM model - developed in his companion paper coauthored with Henriksson - Merton shows that the portfolio manager's global performance, combining her timing and selectivity abilities, expressed in realized returns and denoted $\pi$, can be represented as

$$
\pi_{\mathrm{HM}, \mathrm{o}}=\alpha_{\mathrm{HM}}+\gamma_{\mathrm{HM}} e^{R_{f} \Delta t} P\left(M, \Delta t, e^{R_{f} \Delta t}\right)
$$

where $P\left(M, \Delta t, e^{R_{f} \Delta t}\right)$ is a put with a remaining time to maturity equal to the time interval and strike price of $e^{R_{f} \Delta t}$ written on the market portfolio $M$, whose price is normalized to 1

The reasoning underlying Merton's result is that the portfolio return obtained in equation (1) can be achieved by taking at the same time a long position of $\beta_{\mathrm{HM}}$ in the underlying index and of $\gamma_{\mathrm{HM}}$ in a put on the same index, which will only pay off if the index return is lower than the riskless rate. The remaining amount $1-\beta_{\mathrm{HM}}-\gamma_{\mathrm{HM}}$ is invested (if positive) or borrowed (if negative) at the risk-free rate. The cost of adopting such a strategy is the initial put premium, which is a lump sum amount $P\left(S, \Delta t, e^{R_{f} \Delta t}\right)$ per unit of gamma, and that would need to be capitalized to represent the realized opportunity cost of the strategy versus a linear one.

Ingersoll et al. (2007) adapt (5) to the TM framework and obtain

$$
\pi_{\mathrm{TM}, \mathrm{o}}=\alpha_{\mathrm{TM}}+\gamma_{\mathrm{TM}} e^{2 R_{f} \Delta t}\left(e^{\sigma_{m}^{2} \Delta t}-1\right)
$$

where $R_{f}$ is the continuous interest rate and $\sigma_{m}^{2}$ is the variance of the market portfolio returns. The authors interpret the second term of this expression as the payoff for the fraction $\gamma_{\mathrm{TM}}$ of a 
derivative security that pays the square of the excess market return. At the same time, Ingersoll et al. (2007) concede that this definition of total performance is easily prone to manipulation.

Note that the market beta does not explicitly intervene in any of the three formulations for $\pi$ in the models of Grinblatt and Titman (1994), Bollen and Busse (2004), and Ingersoll et al. (2007). In the Admati et al. (1986) approach, this coefficient represents an output of the model, and it is completely absent from the empirical approach of Bollen and Busse (2004).

There are two major differences between the original Merton (1981) interpretation of the HM model and the transformation proposed by Ingersoll et al. (2007). The first difference is that, once we depart from the Admati et al. (1986) strict optimization framework, the value of $\gamma_{\mathrm{TM}}$ in the TM formulation can be fully independent of the level of $\beta_{\mathrm{TM}}$. A market timer can freely choose her beta and, at the same time, achieve a level of performance that depends on her skill (or luck) in timing the market. In the HM model, this is not the case: the level of $\gamma_{\mathrm{HM}}$ reflects the difference between the high market beta and the low market beta. Therefore the value of $\beta_{\mathrm{HM}}$ is present, albeit implicitly, in equation (5). The second reason for the difference between the two models is that the Merton interpretation rests on the cost of replicating a protective put on the market. The quadratic option proposed in equation (6) is not tradable, and so the reasoning is held within a context of incomplete markets. Below, we follow the very same reasoning as Merton (1981) within the scope of the TM model.

\section{The Treynor and Mazuy model revisited}

We examine the properties of a passive portfolio strategy that would exhibit a pattern of returns similar to equation (2). In the absence of any managerial skill, there is a direct connection between the levels of the beta and gamma coefficients on the one hand, and the intercept of the regression on the other. The difference between the resulting "passive" level of alpha and the actual alpha delivers the performance attributable to the manager. 


\subsection{Option replication in the Treynor and Mazuy model}

Consider a simple self-financing investment strategy consisting of creating a long position in an index with a positive or a negative convexity in returns and lending or borrowing at the risk-free rate. A position involving a long call option written on this index has a positive delta and a positive gamma. Similarly, a position involving a short put option has a positive delta and a negative gamma. In principle, one can find an option whose time-to-maturity and moneyness match the desired sensitivities to the underlying index.

Formally, we can rewrite equation (2) of the actively managed portfolio in terms of total returns:

$$
\begin{aligned}
R_{t}-R_{f} & =\alpha_{\mathrm{TM}}+\beta_{\mathrm{TM}}\left(R_{m t}-R_{f}\right)+\gamma_{\mathrm{TM}}\left(R_{m t}-R_{f}\right)^{2}+\varepsilon_{t} \\
& \Rightarrow R_{t} \simeq \alpha_{\mathrm{TM}}^{\prime}+\beta_{\mathrm{TM}} R_{m t}+\gamma_{\mathrm{TM}} R_{m t}^{2}+\varepsilon_{t}
\end{aligned}
$$

where $\alpha_{\mathrm{TM}}^{\prime}=\alpha_{\mathrm{TM}}+\left(1-\beta_{\mathrm{TM}}\right) R_{f}$ and the second line follows from the fact that $R_{f}^{2} \simeq 0$ and $R_{m t} R_{f} \simeq 0 .^{3}$

It is possible to use options in order to reproduce the return generating pattern of (7). The equation involves positive linear and quadratic sensitivities with respect to the index. Such a portfolio with a positive convexity can be reproduced by a protective put, considering only second-order effects of the option sensitivities (i.e. delta and gamma). To do so, we create a passive portfolio consisting of a series of positions $w_{i}>0$ in $n$ options on the market index, the remainder of the portfolio $\left(1-\sum w_{i}\right)$ being held in a combination $x$ of the risk-free asset and $(1-x)$ of the index. Unlike in the HM model, in which the maturity and moneyness of the option are constrained by the return generating function, the option characteristics are left open. As before, we normalize to 1 the price of the market portfolio at time $t$.

Let $\widetilde{R}_{t}$ be the rate of return of the passively managed portfolio involving only a constant investment in the index, the risk-free asset, and index options. Using the second order Taylor series expansion, the rate of return during the interval $\Delta t$ of the portfolio can be expressed using the

\footnotetext{
${ }^{3}$ For instance, using weekly data over the $1999-2014$ period, the average of $R_{m}^{2}$ has an order of magnitude of $10^{-3}$, while for the average of $R_{f}^{2}$ and $R_{m} R_{f}$ it reduces to $10^{-7}$.
} 
"greeks", i.e. the partial derivatives of the options with respect to selected variables:

$$
\widetilde{R}_{t}=\sum_{i=1}^{n} w_{i}\left(\Delta^{(i)} R_{m t}+\frac{1}{2} \Gamma^{(i)} R_{m t}^{2}+\Theta^{(i)}\right)+\left(1-\sum_{i=1}^{n} w_{i}\right)\left(x R_{f}+(1-x) R_{m t}\right)+o(\Delta t)
$$

where $\tau$ and $\kappa$ are the time-to-maturity and the strike price (expressed as a multiple of the spot price) of the option, respectively, and $\Delta^{(i)} \equiv \frac{\partial F_{i}}{\partial M}, \Gamma^{(i)} \equiv \frac{\partial^{2} F_{i}}{\partial M^{2}}$ and $\Theta^{(i)} \equiv \frac{\partial F_{i}}{\partial t}$ are the option deltas, gammas and thetas. Note that, in the context of constant interest rates and market volatility over the time interval, the remaining greeks (rho and vega) do not show up. The remaining term $o(\Delta t)$ results from the higher orders of the Taylor series expansion and from the potential rho and vega effects.

Besides the residual term, equations (7) and (8) look very similar. The linear and quadratic coefficients are equal if the following set of conditions is satisfied:

$$
\begin{aligned}
\beta_{\mathrm{TM}} & =\sum_{i=1}^{n} w_{i} \Delta^{(i)}+\left(1-\sum_{i=1}^{n} w_{i}\right)(1-x) \\
\gamma_{\mathrm{TM}} & =\frac{1}{2} \sum_{i=1}^{n} w_{i} \Gamma^{(i)}
\end{aligned}
$$

These two identities ensure that the linear and quadratic sensitivities in (7) and (8) are equal. An investor could thus enter an arbitrage portfolio by taking a long position in the active portfolio and a short position in the passive one, with a neutralization of the linear and quadratic systematic risk exposures. Its rate of return is given by:

$$
R_{t}-\widetilde{R}_{t}=\alpha_{\mathrm{TM}}^{\prime}-\sum_{i=1}^{n} w_{i} \Theta^{(i)}-\left(1-\sum_{i=1}^{n} w_{i}\right) x R_{f}+\left(\varepsilon_{t}-o(\Delta t)\right)
$$

in which the last term represents the residual specific risk of the portfolio, to be diversified away.

Naturally, the passive manager's objective is to minimize the cost of replication, i.e. to maximize the constant rate of return retrieved from equation (8) defined as:

$$
\widetilde{\alpha}=\max _{w_{1}^{*}, \ldots, w_{n}^{*}, x^{*}}\left[\sum_{i=1}^{n} w_{i}^{*} \Theta^{(i)}+\left(1-\sum_{i=1}^{n} w_{i}^{*}\right) x^{*} R_{f}\right] .
$$

while simultaneously respecting equations (9) and (10). 
An active portfolio manager only delivers positive performance if she manages to exceed the constant of the best possible replication portfolio $\widetilde{\alpha}$ thanks to a superior intercept $\alpha_{\mathrm{TM}}^{\prime}$. Hence, to assess the performance of the actively managed portfolio reflected in equation (7), we finally subtract the average return of the replicating portfolio:

$$
\begin{aligned}
\widetilde{\pi}_{\mathrm{TM}} & =\bar{R}_{t}-\overline{\widetilde{R}}_{t}=\alpha_{\mathrm{TM}}^{\prime}-\widetilde{\alpha} \\
& =\alpha_{\mathrm{TM}}+\left[1-\beta_{\mathrm{TM}}-\left(1-\sum_{i=1}^{n} w_{i}^{*}\right) x^{*}\right] R_{f}-\sum_{i=1}^{n} w_{i}^{*} \Theta^{(i)}
\end{aligned}
$$

As for the other adjustments proposed in the literature, the global performance of a market timer can be decomposed into the regression alpha plus an adjustment, but this adjustment now explicitly accounts for the portfolio beta, which affects the optimal mix of options and the leverage level in the replicating portfolio.

\subsection{Portfolio performance through replication with a single instrument}

The adjustment proposed in equation (13) involves the selection of a number of options as well as the level of leverage. Even though this adjustment provides the best possible solution to the portfolio replication problem, this measure is not likely to be implemented as a performance measure in practice. There are two reasons for this. First, the design of $\tilde{\pi}_{\mathrm{TM}}$ involves a complex process of selecting different options and mixing them with each other and with the linear exposure in the index in the optimal way. This sophisticated program would require a substantial effort to periodically rebalance the portfolio in order to maintain its optimal character. But even if such an algorithmic procedure were accessible at reasonable cost, there is a second, more fundamental reason for not considering expression (13) as a performance measure: it does not correspond to a passive portfolio replication procedure. In order to assess this cost, a single replication rule must be adopted for the whole period under study, and it must be implementable in a systematic manner. Knowing ex post what the active portfolio manager has done, it is impossible to go back in time and discover what would have been the best mix of derivatives that would replicate the slope and curvature of the portfolio. Rather, it is reasonable to assess that the passive manager who would have obtained the 
same beta and gamma would have chosen one single option, presumably the cheapest one, which could have yielded the same linear and quadratic sensitivities to the benchmark index.

We can also justify the use of a single option in the replicating portfolio as a special case of the general problem described above. If the replicating portfolio is constrained to feature one option and no linear instrument (ETF or future), then the actual replicating cost will be higher. In order to achieve this, the constant of equation (8) must be lower than $\widetilde{\alpha}$ or, equivalently, the estimated portfolio performance must be higher than $\widetilde{\pi}_{\mathrm{TM}}$. This means that the performance measure obtained with a single replicating instrument will be interpreted as the upper bound of the actual portfolio manager's performance. As it is technically possible to replicate her portfolio within more favorable conditions, the estimated performance presumably overestimates the true skill of the market timer.

Using a single option and no linear instrument in the replicating portfolio implies setting $n=1$ and $x=1$. Along the same lines as before, we create a passive portfolio consisting of a long position $w>0$ in a call option on the market index, the remainder of the portfolio $(1-w)$ being held in the risk-free asset. Equation (8) now becomes:

$$
R_{t}^{(\tau, \kappa)}=w\left(\Delta_{\tau, \kappa} R_{m t}+\frac{1}{2} \Gamma_{\tau, \kappa} R_{m t}^{2}+\Theta_{\tau, \kappa}\right)+(1-w) R_{f}+o(\Delta t)
$$

where $\tau$ and $\kappa$ are the time-to-maturity and the strike price (expressed as a multiple of the spot price) of the option, respectively, and $\Delta_{\tau, \kappa} \equiv \frac{\partial C(M, \tau, \kappa)}{\partial M}, \Gamma_{\tau, \kappa} \equiv \frac{\partial^{2} C(M, \tau, \kappa)}{\partial M^{2}}$ and $\Theta_{\tau, \kappa} \equiv \frac{\partial C(M, \tau, \kappa)}{\partial t}$ are the option delta, gamma and theta. The challenge is to choose the right option, then to set its weight for replication purposes.

The linear and quadratic coefficients of equations (7) and (14) are equal if the following set of conditions is satisfied:

$$
\begin{aligned}
\beta_{\mathrm{TM}} & =w \Delta_{\tau, \kappa} \\
\gamma_{\mathrm{TM}} & =w \frac{1}{2} \Gamma_{\tau, \kappa}
\end{aligned}
$$

The system of two equations with three unknowns $\Delta_{\tau, \kappa}, \Gamma_{\tau, \kappa}$ and $w$ is indefinite, as there is a continuum of pairs $(\tau, \kappa)$ that satisfy conditions (15) and (16). The chosen pair minimizes 
the cost of replication, i.e. it maximizes the constant rate of return, which we call $\alpha^{(\tau, \kappa)}=$ $w_{\tau, \kappa} \Theta_{\tau, \kappa}+\left(1-w_{\tau, \kappa}\right) R_{f}$. The solution to this problem is given through the following program:

$$
\begin{aligned}
& \alpha^{\left(\tau^{*}, \kappa^{*}\right)}=\max _{\tau, \kappa}\left(w_{\tau, \kappa} \Theta_{\tau, \kappa}+\left(1-w_{\tau, \kappa}\right) R_{f}\right) \\
& \text { s.t. } \frac{2 \Delta_{\tau^{*}, \kappa^{*}}}{\Gamma_{\tau^{*}, \kappa^{*}}}=\frac{\beta_{\mathrm{TM}}}{\gamma_{\mathrm{TM}}}
\end{aligned}
$$

where $w_{\tau, \kappa} \equiv \frac{\beta_{\mathrm{TM}}}{\Delta_{\tau, \kappa}}>\beta_{\mathrm{TM}}$.

Having established portfolio coefficients $\beta_{\mathrm{TM}}$ and $\gamma_{\mathrm{TM}}$, one has to screen all index call options that respect equation (18). For each eligible pair $(\tau, \kappa)$, there is a corresponding weighting coefficient $w_{\tau, \kappa}$, which allows us to check the equation (17). The optimal pair $\tau^{*}, \kappa^{*}$ is obtained by screening all possible maturities, attaining the corresponding moneyness, and verifying the values of $\alpha^{(\tau, \kappa)}$ in order to obtain the maximum return.

To obtain the excess performance of the active portfolio that yields the returns (7) over the replicating portfolio, we deduct $\alpha^{\left(\tau^{*}, \kappa^{*}\right)}$ :

$$
\begin{aligned}
\pi_{\mathrm{TM}}^{*} & =\alpha_{\mathrm{TM}}^{\prime}-\alpha^{\left(\tau^{*}, \kappa^{*}\right)} \\
& =\alpha_{\mathrm{TM}}+\left(w_{\tau^{*}, \kappa^{*}}-\beta_{\mathrm{TM}}\right) R_{f}-w_{\tau^{*}, \kappa^{*}} \Theta_{\tau^{*}, \kappa^{*}} \\
& =\alpha_{\mathrm{TM}}+\beta_{\mathrm{TM}}\left[\left(\frac{1}{\Delta_{\tau^{*}, \kappa^{*}}}-1\right) R_{f}-\frac{\Theta_{\tau^{*}, \kappa^{*}}}{\Delta_{\tau^{*}, \kappa^{*}}}\right]
\end{aligned}
$$

As $w_{\tau^{*}, \kappa^{*}}>\beta_{\mathrm{TM}}$ and $\Theta_{\tau^{*}, \kappa^{*}}<0$ (Merton, 1981), both terms inside brackets are positive. The first term reflects the interest income saved by the portfolio manager over the replication portfolio. It represents the incremental money market return earned over the passive portfolio, which must be invested in options as a higher proportion of the portfolio than was the case at the original exposure to the market index. The second term features the option theta, as in Merton (1981). This simply translates the loss in the option time value over the passage of time. Because the adjustment for market timing is strictly positive, a passive manager who generates no performance must generate a negative regression intercept $\alpha_{\mathrm{TM}}$. 


\subsection{Interaction of directional and quadratic exposures}

The reasoning underlying the option replication approach can naturally be extended to the cases of negative convexity and/or of negative directional exposure.

The situation of a portfolio manager who negatively times the market involves a negative convexity coefficient $\gamma_{-\mathrm{TM}}<0$ (where index $-\mathrm{TM}$ represents the negative market timer). The analysis is then conducted along similar lines as above. The replicating portfolio with one option involves going short an amount of $-w$ puts on the index and investing $1+w$ in the riskless asset. The performance of the "contrarian" market timer, denoted $\pi_{-\mathrm{TM}}^{*}$, is obtained by the following equation:

$$
\pi_{-\mathrm{TM}}^{*}=\alpha_{-\mathrm{TM}}-\left(w_{\tau^{*}, \kappa^{*}}+\beta_{-\mathrm{TM}}\right) R_{f}+w_{\tau^{*}, \kappa^{*}} \Theta_{\tau^{*}, \kappa^{*}}
$$

The second and third term are both negative. The intercept of the regression corresponding to a neutral performance is positive.

A manager who adopts a negative directional exposure, such as a Short Selling fund, also fits within this framework. To replicate a fund with a negative beta and a positive gamma, one has to use a long position in a put option. If both the portfolio beta and gamma are negative, then a short position in a call option will be adopted.

The situation of a market neutral fund cannot be replicated by a simple strategy involving a call or a put. Indeed, to create an option portfolio with a zero (or a very low) delta and positive or negative gamma, the appropriate strategy is the bottom or the top straddle. The bottom straddle consists of going simultaneously long on a call and a put with the same strike and maturity, respectively. The top straddle involves the reverse (selling a call and a put).

To activate the straddle, the portfolio beta, which is close to zero, is separated into two parts: a long part $\beta_{\mathrm{TM}}^{+}>0$ and a short part $\beta_{\mathrm{TM}}^{-}<0$. To ensure the identical convexity of each option, we set $\gamma_{\mathrm{TM}}=\gamma_{\mathrm{TM}}^{+}+\gamma_{\mathrm{TM}}^{-}$where $\frac{\beta_{\mathrm{TM}}^{+}}{\gamma_{\mathrm{TM}}^{+}}=-\frac{\beta_{\mathrm{TM}}^{-}}{\gamma_{\mathrm{TM}}^{-}}$. Using the same superscripts for the greeks of the call option $(+)$ and of the put option $(-)$, the performance of this non-directional fund is given by:

$$
\pi_{\mathrm{TM}}^{n d}=\alpha_{\mathrm{TM}}+\left(w_{\tau^{*}, \kappa^{*}}^{+}+w_{\tau^{*}, \kappa^{*}}^{-}-\beta_{\mathrm{TM}}\right) R_{f}-w_{\tau^{*}, \kappa^{*}}^{+} \Theta_{\tau^{*}, \kappa^{*}}^{+}-w_{\tau^{*}, \kappa^{*}}^{-} \Theta_{\tau^{*}, \kappa^{*}}^{-}
$$


Table 1 summarizes the kind of option strategy replicating each type of market timing portfolio.

Insert Table 1 here

\subsection{Multi-factor quadratic model}

An actively managed portfolio producing a quadratic pattern of returns such as in (7) is likely to bear a significant specific risk, represented by $\varepsilon_{t}$ in the equation. There is ample evidence that this error term does not behave like white noise. As Detemple et al. (2010) point out, the correlation between the portfolio and market returns in the presence of market timing varies with the level of market returns. This is empirically confirmed by Mattalin et al. (2011). As a result, the specific component $\varepsilon_{t}$ displays ample heteroskedasticity. This could distort the arbitrage argument that is essential to the portfolio replication approach. Therefore, the specification issue has a particular importance in this context. One has to ensure that the return generating process employed reaches a very high explanatory power. The proposed multi-factor extensions of the original market model employed by Treynor and Mazuy are of interest in this regard.

Bollen and Busse (2004) and Krimm et al. (2011) apply the Fama and French (1993) - Carhart (1997) four-factor model to obtain a more precise return-generating process, and they add a single term to reflect the ability to anticipate variations in the market returns. This basic setup has been extended along two dimensions. Chen et al. (2013) apply quadratic terms to all four risk factors, and find evidence of growth timing. Comer (2006) and Comer et al. (2009) extend the four-factor specification to a set of four bond indexes retrieved from the Blake et al. (1993) 6-factor bond model. This overlay enables the authors to capture the potential risk exposures of hybrid funds, with a reported average R-squared value reaching more than $98 \%$ for the two samples they studied.

Lehmann and Modest (1987) provide the foundations for generalizing the TM model to a multifactor setting. Considering that the manager can anticipate the variations in a number $K$ of indexes, the authors propose the addition of the same number of squared returns to the regression, but also the addition of all the two-by-two interaction terms. This would leave us with $K(K-1) / 2$ market 
timing terms to compute, with presumably a high number of insignificant explanatory variables, inducing issues of overspecification. In his 8-factor model (4 for stocks and 4 for bonds), Comer (2006) defends a more parsimonious solution. He discards all the interaction terms, and considers two market timing factors: one for the stock market and one for the bond market.

Our portfolio replicating approach carries a simpler multi-factor extension. Consider that the linear return generating specification features $K$ risk factors, but that only a subset $L \leq K$ are prone to a market timing behavior. For each of these $L$ factors, we can isolate the linear and quadratic sensitivities and apply the same reasoning as in the previous section. For each underlying index, the weight to be invested in options is first determined. Once all the weights are set, they are summed up with the linear betas on the $K-L$ factors with no market timing, and the remainder of the portfolio value is invested in the risk-free asset. Formally, the model is written as:

$$
R_{t} \simeq \alpha+\left(1-\sum_{i=1}^{K} \beta_{i}\right) R_{f}+\sum_{i=1}^{K} \beta_{i} R_{i t}+\sum_{i=1}^{L} \gamma_{i} R_{i t}^{2}+\varepsilon_{t}
$$

where, as in equation (7), the terms $R_{f}^{2}$ and $R_{i t} R_{f}$ are assumed to be negligible. The associated market timing adjusted performance is computed as:

$$
\pi^{*}=\alpha+\sum_{i=1}^{L}\left(w_{\tau_{i}^{*}, \kappa_{i}^{*}}-\beta_{i}\right) R_{f}-\sum_{i=1}^{L} w_{\tau_{i}^{*}, \kappa_{i}^{*}} \Theta_{\tau_{i}^{*}, \kappa_{i}^{*}}
$$

which is a straight generalization of equation (19) to a multi-index model. Note that we do not require any correlation structure between the risk factors, as each replicating portfolio is considered in isolation.

\section{Option rebalancing and cost issues}

The portfolio replicating approach of the TM quadratic return generating process creates two potential problematic issues. The first relates to the risk of discrete rebalancing, which may induce a drift in the linear and quadratic sensitivities of the replicating portfolio to the underlying of the option, reducing the mimicking properties of the portfolio. The second is linked to the objective of finding 
the cheapest replicating option, whose empirical behavior (if maturity is too low, for instance) could become too remote from the market timing portfolio even though the beta and gamma constraints might be formally met. We discuss these two issue below.

\subsection{Systematic rebalancing risk}

Equation (8) holds when portfolio rebalancing occurs continuously. When returns are measured on a discrete basis (from one day to two months), for instance, the risk of the hedged portfolio can become substantial (Gilster, 1997). Even for relatively short frequencies (one day or one week), Gilster shows that the rebalancing risk of a hedged position is usually significant.

Nevertheless, the issue of the replication of the market timer portfolio is less about the extent of the rebalancing risk than about its systematic character, i.e. its impact on the regression beta and gamma of the replicating portfolio. Gilster $(1990,1997)$ documents that replicating portfolios for near-to-maturity options, when rebalanced in discrete-time, exhibit significant systematic risk with the underlying index returns. Considered within the TM quadratic regression framework, the beta would not simply correspond to the product $w \Delta_{\tau, \kappa}$ as in equation (9), but would be greater. Part of the bias could be corrected for through the quadratic beta that also explains the replicating portfolio returns, thereby reflecting the gamma risk emphasized by Gilster (1997). Thus, with the specification of equation (14), the rebalancing risk would mostly refer to a third-order effect.

To check the magnitude of this bias, it is necessary to ensure that (i) the slope and convexity of the sensitivity of the replicating portfolio match the beta and gamma of the TM regression (fitting constraint), and (ii) the ex-post cost of the replication is close to the ex-ante estimation (unbiasedness constraint).

To respect the first constraint, the strike price and time-to-maturity of the cheapest-to-replicate option would need to respect some tolerance regarding the quality of the quadratic fit regarding the individual coefficients. This translates into the need to supplement the optimization problem (17) 
with two additional constraints:

$$
\begin{aligned}
& \left|\beta_{\mathrm{TM}}-\beta^{\left(\tau^{*}, \kappa^{*}\right)}\right| \leq \operatorname{tol}_{\beta} \\
& \left|\gamma_{\mathrm{TM}}-\gamma^{\left(\tau^{*}, \kappa^{*}\right)}\right| \leq \operatorname{tol}_{\gamma}
\end{aligned}
$$

where $\beta^{\left(\tau^{*}, \kappa^{*}\right)}, \gamma^{\left(\tau^{*}, \kappa^{*}\right)}$ and $\eta_{t}$ are obtained by the reverse quadratic regression of the replicating portfolio returns on the underlying index: ${ }^{4}$

$$
R_{t}^{\left(\tau^{*}, \kappa^{*}\right)}=c^{\left(\tau^{*}, \kappa^{*}\right)}+\beta^{\left(\tau^{*}, \kappa^{*}\right)} R_{m t}+\gamma^{\left(\tau^{*}, \kappa^{*}\right)} R_{m t}^{2}+\eta_{t}
$$

Imposing restrictions on the significance of the reverse quadratic regression compared to the TM specification would be superfluous. The TM model represents the return generating process of an actively managed portfolio, which potentially mixes asset selection and market timing skills. If the manager places strong emphasis on selectivity, the residual term $\varepsilon_{t}$ of the quadratic regression will presumably be volatile, and the specific risk will be high. ${ }^{5}$ As the reverse quadratic regression reproduces a mere fitting exercise on passive portfolio returns, there is no expected connection with the significance of the original market timing model.

Regarding the second (unbiasedness) constraint, the reverse regression provides a quality check of the ex-post (actual) cost of the option replicating approach. The difference between equations(27) and $(8)$ taken at point $\left(\tau^{*}, \kappa^{*}\right)$ yields the following inequality:

$$
\left|c^{\left(\tau^{*}, \kappa^{*}\right)}-\alpha^{\left(\tau^{*}, \kappa^{*}\right)}\right| \leq \operatorname{tol}_{\beta}\left|\bar{R}_{m}\right|+\operatorname{tol}_{\gamma} \bar{R}_{m}^{2}=\operatorname{tol}_{\alpha}
$$

If the linear quadratic coefficients are arbitrarily close to the original TM values, then the constant of the regression $c^{\left(\tau^{*}, \kappa^{*}\right)}$ will also approach the theoretical value of the constant rate of return $\alpha^{\left(\tau^{*}, \kappa^{*}\right)}$. The difference between $c^{\left(\tau^{*}, \kappa^{*}\right)}$ and $\alpha^{\left(\tau^{*}, \kappa^{*}\right)}$ is an empirical matter. Expression $\alpha^{\left(\tau^{*}, \kappa^{*}\right)}$ represents an ex-ante cost of replicating the market timer's portfolio with options, and

\footnotetext{
${ }^{4}$ As they only involve individual parameter values, the constraints are compatible with the multi-factor specification (23).

${ }^{5}$ Nothing in our setup precludes the use of the appraisal ratio to assess the risk-adjusted performance of the manager. This simply entails replacing the regression alpha with expression (24) at the numerator.
} 
corresponds to the original Merton (1981) interpretation. After the option replicating strategy has been systematically implemented, the outcome of regression (27) depends on the realizations of the index returns. This may induce deviations from the effects foreseen by the Taylor series expansion. For instance, a very large outlier may have occurred during the estimation period. Effects of a higher order than two, captured neither by the delta nor by the gamma, might then become significant in the option returns. These effects impact the estimates of $\beta^{\left(\tau^{*}, \kappa^{*}\right)}$ and $\gamma^{\left(\tau^{*}, \kappa^{*}\right)}$ in the quadratic fitting equation. The regression intercept $c^{\left(\tau^{*}, \kappa^{*}\right)}$ represents the ex post return of the option portfolio.

\subsection{Cost-minimizing option}

Implementing a strategy that consists of replicating a portfolio with a single long call option involves a careful selection of this option. As the underlying asset is determined by the selection of the index in the TM model, the choice collapses to setting the moneyness $\kappa$ and the time-to-maturity $\tau$ of the option. The contract must respect a constraint, namely the target level of the ratio of the delta over its gamma in equation (18). Then, amongst all eligible options, the best one is the option that minimizes the cost of replication, i.e. that maximizes expression $w_{\tau, \kappa} \Theta_{\tau, \kappa}+\left(1-w_{\tau, \kappa}\right) R_{f}$. The function to maximize depends on the pair $(\tau, \kappa)$ through the option theta, but also through the weight invested in the option in the passive portfolio $w_{\tau, \kappa} \equiv \frac{\beta_{\mathrm{TM}}}{\Delta_{\tau, \kappa}}$.

The partial derivatives of delta and gamma with respect to time are usually called Charm and Color, respectively (Garman, 1992). In the Black-Scholes-Merton world, they bear an analytical form and their behavior is well-known. Unfortunately, even in such a controlled environment, their signs are erratic. Haug (2003) presents an example where the Charm is negative for ITM and positive for OTM calls, but at the same time the Color is negative for the near ATM and positive for the far OTM or ITM options. Overall, the evolution of the ratio of delta over alpha over time (and so their derivative with respect to time-to-maturity) is indeterminate.

We illustrate in Figure 1 the sets of parameters that reach different target values of the ratio

$\frac{\beta_{\mathrm{TM}}}{\gamma_{\mathrm{TM}}}=\frac{2 \Delta_{\tau, \kappa}}{\Gamma_{\tau, \kappa}}$ for options that are priced under the Black-Scholes-Merton model. We take as inputs 
the average 3-month T-Bill rate and average volatility of the S\&P500 weekly returns over the Jan. 1999 - Sept. 2008 period, namely $0.066 \%$ and 2.289\%, respectively. The plotted contour lines correspond to the target values of this ratio taking multiples of $1 / 3$, ranging from 0 to 3 . The ratio represents the fraction of beta over gamma in the TM model. As the average market beta is equal to one, we span in principle values of gamma starting from $1 / 3$ onwards. We set the range of maturities to 1 to 52 weeks, and the range of moneyness ratios from 0.80 to 1.00 .

\section{Insert Figure 1 here}

Interpreting the ratio $\frac{2 \Delta_{\tau, \kappa}}{\Gamma_{\tau, \kappa}}$ as a reverse indicator of curvature, Figure 1 shows that only ITM options (i.e. whose strike $\kappa<1$ ) provide potentially meaningful convexity. For long maturity options, the progression of the ratio remains gradual. With a one-year maturity option, it takes a moneyness of ca. $90 \%$ to obtain a beta equal to half the option gamma or, by identity, a TM beta equal to its gamma. On the other hand, the ratio evolves very quickly with short maturity options. When the maturity approaches one week, i.e. when the lowest maturity is not lower than the frequency of returns estimation, the ratio becomes huge when the moneyness reduces to lower than $93 \%$.

The next step of the analysis is to find out the cheapest-to-replicate option among the options that respect a target ratio value. Following the same example as above, we set $\beta_{\mathrm{TM}}=1$ and let the value of $\frac{\beta_{\mathrm{TM}}}{\gamma_{\mathrm{TM}}}$ be equal to 2 (blue line), 1 (red line) and 0.67 (green line). These values correspond, respectively, to the contour lines between the dark and light green regions (ratio $=2$ ), sky and light blue regions (ratio $=1$ ) and light and dark blue regions (ratio $=0.67$ ) in Figure 1 . The $\gamma_{\mathrm{TM}}$ equals $0.5,1.0$ and 1.5, which are reasonable values for a market timer as shown later in our empirical study. For each feasible pair $(\tau, \kappa)$, we compute the cost of the option replicating portfolio $-\alpha^{(\tau, \kappa)}=-w_{\tau, \kappa} \Theta_{\tau, \kappa}-\left(1-w_{\tau, \kappa}\right) R_{f}$. The lower this cost, the cheaper it is to replicate the option.

Insert Figure 2 here

The replication cost increases with the level of $\gamma_{\mathrm{TM}}$. This is the natural consequence of increasing the convexity of the portfolio payoff, which is done at the expense of the option theta. The com- 
parison of the three lines shows quite small differences between the patterns of the cost function. For $\gamma_{\mathrm{TM}}=0.5$ (blue line), the cost increases from $1.420 \%$ to $1.613 \%$. The start and end points are $2.853 \%$ and $3.319 \%$ for $\gamma_{\mathrm{TM}}=1$, and $4.293 \%$ and $5.105 \%$ for $\gamma_{\mathrm{TM}}=1.5$, respectively. Thus, the cost increases slightly less than proportionally with the value of gamma and with maturity. ${ }^{6}$

In all the illustrated cases, the option replication cost increases with option maturity. Even though this result cannot be generalized (because option Charm and Color have indefinite signs), our realistic example shows that this scenario can happen. This means that, in the absence of any constraint on specific or approximation risk control, the cheapest-to-replicate option might have a maturity of one period. As it matches the frequency of returns computation, the option-based portfolio produces the same returns as in the HM model, and is unlikely to be adequately estimated using the TM specification in the reverse regression. Hence, our case illustrates the need to assign constraints (25) and (26) for the reverse regression of option returns.

\section{The choice of the replicating option}

The goal of this section is to identify the most satisfactory trade-off between the regression fit and the accuracy of the intercept.

As indicated in the previous section, the option choice without constraints might realistically indicate that the shorter the time to maturity, the cheaper the option replication. The reverse quadratic regression (27) restricts the feasibility of maturity reduction because the quality of the fit naturally deteriorates as the option maturity decreases. The goal of this subsection is to detect, within the same setup as in the previous example, the range of option maturities for which the approximation error induced by the Taylor series expansion is "acceptable", i.e. it falls within the tolerance bounds for the alpha, beta and gamma retrieved from the quadratic regression.

We set portfolio beta as equal to one and adopt the same set of TM gammas as before, namely 0.5, 1.0 and 1.5. The sample period is Jan. 1999 - Sept. 2008 and we create portfolios with a

\footnotetext{
${ }^{6}$ For a given value of gamma, the cost rises proportionally to the level of beta regardless of the maturity.
} 
quadratic exposure on the S\&P500 index. In order to ensure the correspondence between the Black-Scholes option prices and the behavior of the time series of index returns, we posit a flat weekly volatility of $2.375 \%$. By using the sample standard deviation of returns in option prices, we avoid introducing a pricing bias in the estimation of regression (27). For each feasible pair $(\tau, \kappa)$, a portfolio is constituted every week by investing a weight $w_{\tau, \kappa}$ in the option at a price $C(M, \tau, \kappa)$ and $\left(1-w_{\tau, \kappa}\right)$ in the risk-free asset. The following week, the option is sold at a price $C\left(M, \tau-1, \frac{\kappa}{\left(1+R_{m}\right)}\right)$, the risk-free return is booked on the remaining part, and the portfolio is rebalanced. We estimate the reverse quadratic regression by applying the TM specification to the returns of this portfolio.

Figure 3 reports the evolution of the difference between the intercept of the reverse regression and the analytical portfolio alpha, i.e. $c^{(\tau, \kappa)}-\alpha^{(\tau, \kappa)}$ (Figure 3a), the reverse regression beta (Figure $3 \mathrm{~b}$ ), and the reverse regression gamma (Figure 3c), as a function of the time-to-maturity from 1 to 30 weeks. The values for $\gamma_{\mathrm{TM}}=0.5,1.0$ and 1.5 are printed in green, red and blue, respectively.

\section{Insert Figures 3a, 3b and 3c here}

While the analytical replicating cost $-\alpha^{(\tau, \kappa)}$ increases with the option maturity, Figure 3a shows that the regression intercept $c^{(\tau, \kappa)}$ becomes closer to zero as time-to-expiration rises. Because of the poor regression fit for near-maturity options, $c^{(\tau, \kappa)}$ starts at a very negative level (from $-6.24 \%$ to $-8.58 \%$ ). As the regression significance level increases with maturity, the intercept gradually approaches zero. In the cases illustrated in Figure 3, the two functions intersect at maturities equal to 23 weeks $\left(\gamma_{\mathrm{TM}}=0.5\right), 14.5$ weeks $\left(\gamma_{\mathrm{TM}}=1.0\right)$ and 10.5 weeks $\left(\gamma_{\mathrm{TM}}=1.5\right)$. Figures $3 \mathrm{~b}$ and $3 \mathrm{c}$ indicate that the convergence of the coefficients for the linear and quadratic term asymptotically converge to their theoretical values. The speed of convergence typically decreases after a 5 -week maturity. The linear coefficient $\left(\beta^{(\tau, \kappa)}\right)$ remains very close to its target value of 1 , with the distance becoming lower than 0.01 when the maturity exceeds 20 weeks. As expected from the imperfect fit of the second order approximation, the value of $\gamma^{(\tau, \kappa)}$ remains more remote. The coefficient estimate remains systematically upwardly biased with respect to its target value. This results from the small variability of the observed squared market returns $\left(\sigma\left(R_{m}^{2}\right)=0.90 \%\right.$ on a yearly basis $)$ as compared 
to the returns $\left(\sigma\left(R_{m}\right)=17.13 \%\right)$. In this OLS setup, the smaller variation in the independent variable translates into a larger standard deviation in the estimated coefficient.

To obtain a more rigorous analysis of the set of option maturities that support reasonable coefficient values for the reverse quadratic regression, we apply equations (25) and (26) to our data set. To reflect the volatility levels of the independent variables, the tolerance levels are adjusted by setting $t_{0} l_{\beta}=\theta / \hat{t}_{R_{m}}$ and $t o l_{\gamma}=\theta / \hat{t}_{R_{m}^{2}}$ for different values of a constant $\theta$. This yields naturally $\operatorname{tol}_{\alpha}=\theta\left(\frac{\left|\bar{R}_{m}\right|}{\hat{t}_{R_{m}}}+\frac{\bar{R}_{m}^{2}}{\hat{t}_{R_{m}^{2}}^{2}}\right)$ by applying equation (28). We set $\theta$ to $0.006,0.004$ and 0.002 . These values are chosen so as to produce usable maturity intervals and to analyze how they shrink as the tolerance level decreases. The results are displayed in Table 2.

\section{Insert Table 2 here}

For each value of $\Gamma_{\tau, \kappa}$ and $\theta$, the table reports the maturity intervals that respect the tolerance level for each parameter. The last row displays the intersection between these intervals. Interestingly, the intervals become thinner as the convexity of returns diminishes. The regression intercept yields the most severe constraint on the upper bound of the interval because, as shown in Figure 3a, the reverse regression intercept tends to become too large for longer maturities, while its theoretical value is assumed to decrease. For $\theta=0.006$, a large set of option maturities is acceptable, while the interval becomes an empty set for too low convexity and tolerance levels $\left(\Gamma_{\tau, \kappa}=0.5\right.$ and $\left.\theta=0.002\right)$. Overall, maturities between 18.9 and 26.8 weeks comply with most intervals: any maturity fits for $\Gamma_{\tau, \kappa}=1.5$, all from 24.8 to 26.8 weeks for $\Gamma_{\tau, \kappa}=1.0$, and from 18.9 to 23.8 weeks for $\Gamma_{\tau, \kappa}=0.5$.

To summarize our results, the simulations indicate that, for reasonable values of the option convexity gamma of the replicating option, maturities of around six months induce the best match between the regression results and the Taylor series expansion of the option replicating strategy. 


\section{Conclusion}

There is a clear isomorphism between the returns of a market timing fund and the pattern of a protective put or a covered call. Nevertheless, when performance measurement is involved, this isomorphism has only been convincingly translated in the Henriksson and Merton (1981) model in a companion paper by Merton (1981). Even though the Treynor and Mazuy (1966) approach remains the other major regression-based model for the measurement of a market timer's performance, the literature has nevertheless produced only approximate performance corrections. Except in the work of Detemple et al. (2010), who theoretically adjust performance for the fund's specific risk, none of the solutions proposed so far explicitly accounts for the fund's linear sensitivity (beta) with respect to the benchmark portfolio. This shortcoming can only lead to the adjustment being imperfect.

Our paper goes back to basics. Starting from the quadratic return specification underlying the TM model, we identify a passive replicating portfolio with cash and options. The intuition is straightforward: in a mean-variance world where options are priced using the Black-Scholes-Merton formula, any passive portfolio manager could achieve this pattern. A manager's performance is thus reflected in the difference between the regression's alpha and the replicating cost of her portfolio.

Starting from this rather simple idea, we have had to overcome two issues. The first relates to the way to revert from the replicating portfolio to the fund's returns. The resulting rebalancing risk is largely alleviated by the quadratic character of the TM equation. The second issue relates to the identification of the cheapest option; we demonstrate how this can be achieved by choosing the option moneyness and maturity. No serious hindrance seems to endanger the practicability of the replication approach. When returns are measured on a weekly basis, option maturities between one quarter and one semester seem to provide a reasonable trade-off between the cost and the effectiveness of the replicating strategy.

This research opens up the way to establishing a fresh view of the timing skills of managers active in the mutual fund and the hedge fund industry. With the methodological framework developed in this paper, we hope to provide new avenues for research in the performance of market timers. 


\section{References}

Admati, A., Bhattacharya, S., Pfleiderer, P. and Ross, S. (1986). "On Timing and Selectivity",. The Journal of Finance, Vol. 41 No. 3, pp. 715-32.

Agarwal V. and Naik, N.Y. (2004), "Risks and Portfolio Decisions Involving Hedge Funds", Review of Financial Studies, Vol. 17 No. 1, pp. 63-98.

Becker, C., Ferson, W. E., Myers, D. H. and Schill, M. J. (1999). "Conditional market timing with benchmark investors",. Journal of Financial Economics, Vol. 52 No. 1, pp. 119-148.

Blake, C., Elton, E. and Gruber, M. (1993). "The Performance of Bond Mutual Funds",. The Journal of Business, Vol. 66 No. 3, pp. 71-403.

Bollen, N. P. B. and Busse, J. A. (2004). "Short-Term Persistence in Mutual Fund Performance",. Review of Financial Studies, Vol. 18 No. 2, pp. 569-597.

Carhart, M. (1997). "On Persistence in Mutual Fund Performance", The Journal of Finance, Vol. 52 No. 5 , pp. 57-82.

Chen, L.-W., Adams, A. and Taffler, R. (2013), "What Style-Timing Skills do Mutual Fund "Stars" Possess?", Journal of Empirical Finance, Vol. 21 No. C, pp. 156-173 .

Chen, Y. and Liang, B. (2007), "Do Market Timing Hedge Funds Time the Market", Journal of Financial and Quantitative Analysis, Vol. 42 No. 4, pp. 827-856.

Comer, G. (2006). "Hybrid Mutual Funds and Market Timing Performance", The.Journal of Business, Vol. 79 No. 2, pp. 771-97.

Comer, G., Larrymore, N. and Rodriguez, J. (2009). "Controlling for fixed income exposure in portfolio evaluation: Evidence from hybrid mutual funds",. Review of Financial Studies, Vol. 22 No. 2, pp. 481-507.

Detemple, J.B., Garcia, R. and Rindisbacher, M. (2010), "Optimal Portfolio Allocations with Hedge Funds". Working paper, EDHEC Business School.

Elton, E., Gruber, M. and Blake, C. (2011). "An Examination of Mutual Fund Timing Ability Using Monthly Holdings Data",. Review of Finance, Vol. 16 No. 3, pp. 619-645. 
Fama, E. and French, K. (1993). "Common Risk Factors in the Returns on Stocks and Bonds",. Journal of Financial Economics, Vol. 33 No. 1, pp. 3-56.

Fung, W. and Hsieh, D. A. (2001), "The Risk in Hedge Fund Strategies: Theory and Evidence from Trend Followers",. Review of Financial Studies, Vol. 14 No. 2, pp. 313-341.

Garman, M. (1992). "Charm School",. Risk Magazine, Vol. 5 No. 7, pp. 53-56.

Gilster, J. E., Jr. (1990). "The Systematic Risk of Discretely Rebalanced Option Hedges",. Journal of Financial and Quantitative Analysis, Vol. 25 No. 4, pp. 507-516.

Gilster, J. E., Jr. (1997). "Option Pricing Theory: Is 'Risk-Free' Hedging Feasible?", Financial Management, Vol. 26 No. 1, pp. 91-105.

Grinblatt, M. and Titman, S. (1994). "A study of monthly mutual fund returns and performance evaluation techniques",. Journal of Financial and Quantitative Analysis, Vol. 29 No. 3, pp. 419444.

Haug, E.G. (2003). "Know Your Weapon, Part 1", Wilmott Magazine, May, pp. 49-57.

Huang, J., Sialm, C. and Zhang, H. (2011). "Risk Shifting and Mutual Fund Performance",. Review of Financial Studies, Vol. 24 No. 8, pp. 2575-2616.

Henriksson, R. D. and Merton, R. C. (1981). "On Market Timing and Investment Performance. II. Statistical Procedures for Evaluating Forecasting Skills",.The Journal of Business, Vol. 54 No. 4, pp. 513-33.

Ingersoll, J., Spiegel, M., Goetzmann, W. and Welch, I. (2007). "Portfolio Performance Manipulation and Manipulation-proof Performance Measures",. Review of Financial Studies, Vol. 20, No. 5, pp. $1503-1546$.

Jagannathan, R. and Korajczyk, R. (1986). "Assessing the Market Timing Performance of Managed Portfolios",. The Journal of Business, Vol. 59 No. 2, pp. 217-35.

Jensen, M. J. (1968). "The Performance of Mutual Funds in the Period 1945-1964", The Journal of Finance, Vol. 23 No. 2, pp. 389-416.

Jiang, G. J., Yao, T. and Yu, T. (2007). "Do mutual funds time the market? Evidence from port- 
folio holdings",. Journal of Financial Economics, Vol. 86 No. 3, pp. 724-758.

Kaplan, S. N. and Sensoy, B. A. (2005). "Do Mutual Funds Time their Benchmarks?" Working Paper, University of Chicago - Booth School of Business

Krimm, S, Scholz, H. and Wilkens, M. (2011). "Selection, timing and total performance of equity funds: Wasting time measuring timing". Working paper, Catholic University of EichstättIngolstadt.

Kryzanowski, L., Lalancette, S. and To, M. C. (1997). "Performance attribution using an APT with prespecified macrofactors and time-varying risk premia and betas",. Journal of Financial and Quantitative Analysis, Vol. 32 No. 2, pp. 205-224.

Lee, C-F. and Rahman, S. (1990). "Market Timing, Selectivity, and Mutual Fund Performance: An Empirical Investigation",. The Journal of Business, Vol..63 No. 2, pp. 261-278.

Lehmann, B. and Modest, D. (1987). "Mutual fund performance evaluation: A comparison of benchmarks and benchmark comparisons",.The Journal of Finance, Vol. 42 No. 2, pp. 233-65.

Mattalin, J.C., Moreno, D. and Rodriguez, R. (2011). "Why is timing perverse?" Working paper, University Carlos III.

Merton, R. C. (1981). "On Market Timing and Investment Performance. I. An Equilibrium Theory of Value for Market Forecasts",.The Journal of Business, Vol. 54 No. 3, pp. 363-406.

Rodriguez, J. (2014) "The forecasting ability of world mutual funds", Studies in Economics and Finance, Vol. 31, pp. 130-140

Sharpe, W. F. (1966). "Mutual fund performance",.The Journal of Business, Vol. 39, pp. 119-138. Treynor, J. L, (1965). "How to rate management investment funds", Harvard Business Review, Vol. 43 (Jan/Feb), pp. 63-75.

Treynor, J.L. and Mazuy, K. (1966). "Can Mutual Funds Outguess the Market?", Harvard Business Review, Vol. 44 (July-August), pp. 131-136. 


\section{Figures}

Figure 1: Target ratio $\frac{\beta_{\mathrm{TM}}}{\gamma_{\mathrm{TM}}}=\frac{2 \Delta_{\tau, \kappa}}{\Gamma_{\tau, \kappa}}$ as a function of option moneyness and maturity

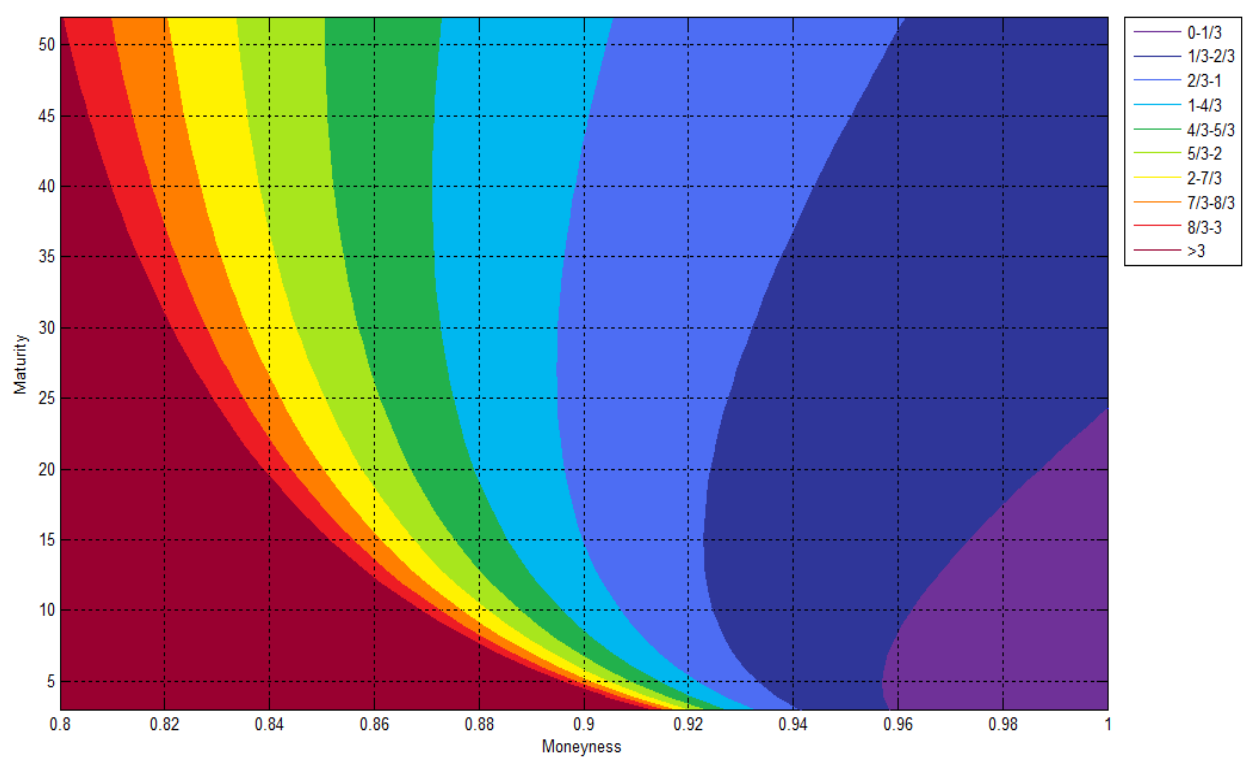

This figure plots the contour lines of the target ratio $\frac{\beta_{\mathrm{TM}}}{\gamma_{\mathrm{TM}}}=\frac{2 \Delta_{\tau, \kappa}}{\Gamma_{\tau, \kappa}}$ as a function of the option moneyness (horizontal axis, expressed as a fraction of the spot underlying price), and maturity (vertical axis, in weeks). We use the Black-Scholes-Merton option pricing formula with the inputs of the average 3-month US Treasury Bill rate and the average volatility of the market index between January 1999 and September 2008, which are set to a weekly value of $0.066 \%$ and $2.289 \%$, respectively. The bottom left area corresponds to values of the ratio above 3 . 
Figure 2: Evolution of $\alpha^{(\tau, \kappa)}$ as a function of option maturity

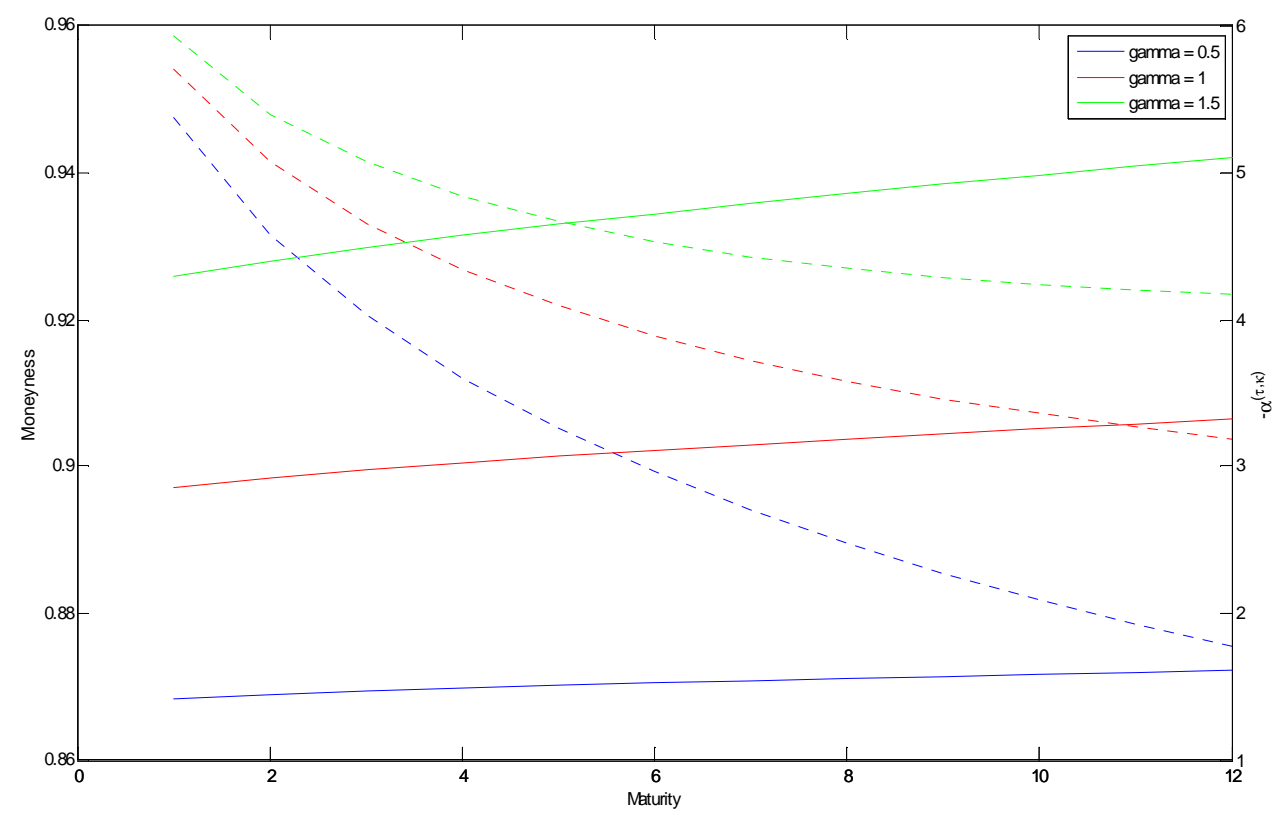

This figure reports $-\alpha^{(\tau, \kappa)}=-w_{\tau, \kappa} \Theta_{\tau, \kappa}-\left(1-w_{\tau, \kappa}\right) R_{f}$ in percentage terms, annualized, as a function of option maturity for $\beta_{\mathrm{TM}}=1$ and a target value of $\frac{\beta_{\mathrm{TM}}}{\gamma_{\mathrm{TM}}}=\frac{2 \Delta_{\tau, \kappa}}{\Gamma_{\tau, \kappa}}$ equal to 2 (blue line), 1 (red line) and 0.67 (green line). The values of $-\alpha^{(\tau, \kappa)}$ are reported on the right axis. The dotted lines represent the corresponding option moneyness expressed as a fraction of the spot underlying price, whose values are reported on the left axis. 
Figure 3: Evolution of $c^{(\tau, \kappa)}, \beta^{(\tau, \kappa)}$ and $\gamma^{(\tau, \kappa)}$ as a function of option maturity

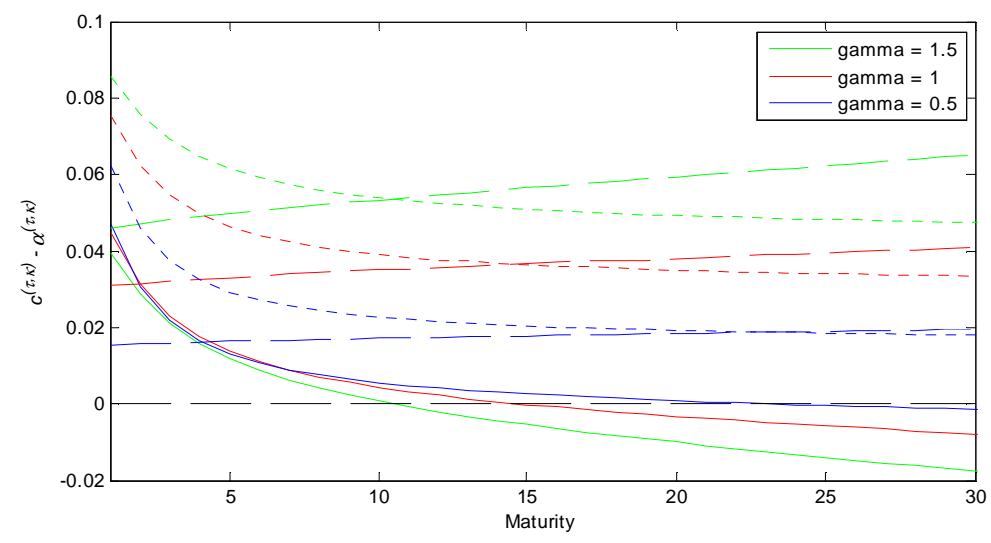

Figure 3a: evolution of $c^{(\tau, \kappa)}-\alpha^{(\tau, \kappa)}$

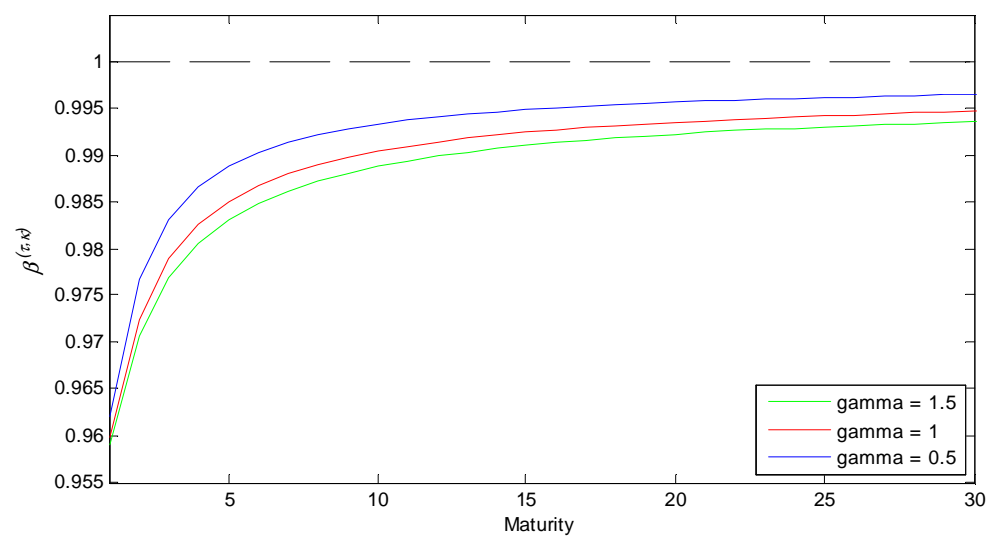

Figure 3b: evolution of $\beta^{(\tau, \kappa)}$

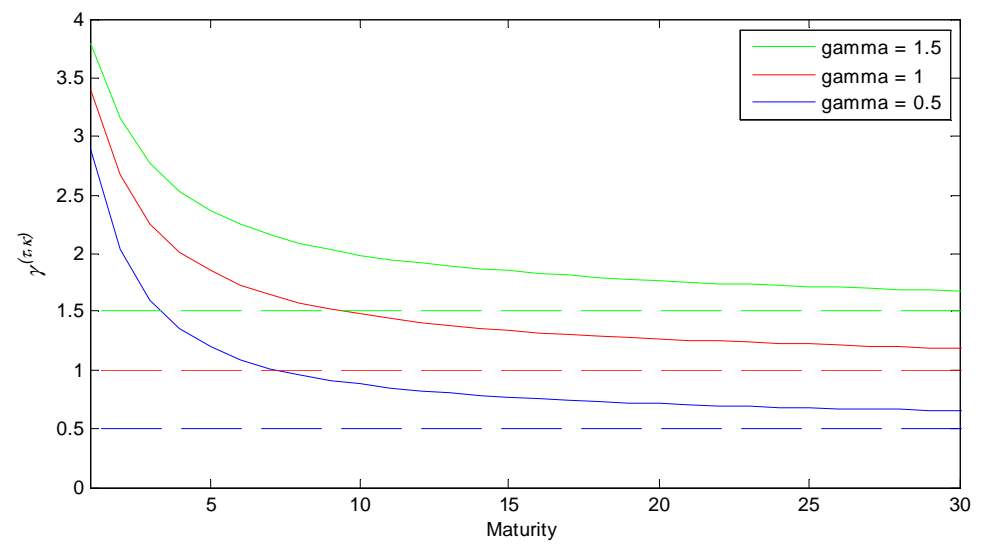

Figure 3c: evolution of $\gamma^{(\tau, \kappa)}$

This figure reports the values of the regression parameters $R_{t}^{(\tau, \kappa)}=c^{(\tau, \kappa)}+\beta^{(\tau, \kappa)} R_{m t}+\gamma^{(\tau, \kappa)} R_{m t}^{2}+$ 
$\eta_{t}$ as a function of option maturity and corresponding moneyness for $\beta_{\mathrm{TM}}=1$ and a target value of $\frac{\beta_{\mathrm{TM}}}{\gamma_{\mathrm{TM}}}=\frac{2 \Delta_{\tau, \kappa}}{\Gamma_{\tau, \kappa}}$ equal to 2 (blue line), 1 (red line) and 0.67 (green line). In Figure 3a, we also report $-\alpha^{(\tau, \kappa)}=-w_{\tau, \kappa} \Theta_{\tau, \kappa}-\left(1-w_{\tau, \kappa}\right) R_{f}$ (thin-dotted line) and the difference $c^{(\tau, \kappa)}-\alpha^{(\tau, \kappa)}$ (solid line). In this figure, the values are shown in percentage terms. In Figures $3 \mathrm{~b}$ and $3 \mathrm{c}$, the thick-dotted lines represent the corresponding target values. 


\section{Tables}

Table 1: Synthesis of option replication strategies

\begin{tabular}{cccc}
\hline \hline & & \multicolumn{2}{c}{ Quadratic exposure } \\
& & $\gamma_{\mathrm{TM}}>0$ & $\gamma_{\mathrm{TM}}<0$ \\
\cline { 3 - 4 } Directional & $\beta_{\mathrm{TM}}>0$ & Long call & Short put \\
exposure & $\beta_{\mathrm{TM}} \simeq 0$ & Bottom straddle & Top straddle \\
& $\beta_{\mathrm{TM}}<0$ & Long put & Short call \\
\hline \hline
\end{tabular}

This table represents the types of strategy involving options that replicate all possible patterns of the TM regression. 
Table 2: Maturity ranges with tolerance levels

\begin{tabular}{ccccc}
\hline Gamma & Criterion & $\theta=0.6 \%$ & $\theta=0.4 \%$ & $\theta=0.2 \%$ \\
\hline$c^{(\tau, \kappa)}-\alpha^{(\tau, \kappa)}$ & {$[3.26,33.1]$} & {$[4.66,23.8]$} & {$[6.84,16.1]$} \\
$\Gamma_{\tau, \kappa}=0.5$ & $\beta^{(\tau, \kappa)}$ & $\geq 1.51$ & $\geq 2.96$ & $\geq 9.24$ \\
& $\gamma^{(\tau, \kappa)}$ & $\geq 6.98$ & $\geq 11.2$ & $\geq 23.8$ \\
$\alpha \cap \beta \cap \gamma$ & {$[\mathbf{6 . 9 8}, \mathbf{3 3 . 1}]$} & {$[\mathbf{1 1 . 2 , 2 3 . 8}]$} & $\varnothing$ \\
\hline$c^{(\tau, \kappa)}-\alpha^{(\tau, \kappa)}$ & $\geq 3.60$ & {$[5.22,44.7]$} & {$[8.31,26.8]$} \\
$\Gamma_{\tau, \kappa}=1.0$ & $\beta^{(\tau, \kappa)}$ & $\geq 1.41$ & $\geq 2.65$ & $\geq 7.22$ \\
& $\gamma^{(\tau, \kappa)}$ & $\geq 6.71$ & $\geq 10.9$ & $\geq 24.8$ \\
$\alpha \cap \beta \cap \gamma$ & $\geq 6.71$ & {$[\mathbf{1 0 . 9}, \mathbf{4 4 . 7}]$} & {$[\mathbf{2 4 . 8} \mathbf{2 6 . 8}]$} \\
\hline$c^{(\tau, \kappa)}-\alpha^{(\tau, \kappa)}$ & $\geq 3.37$ & $\geq 4.95$ & $\geq 8.87$ \\
$\beta^{(\tau, \kappa)}$ & $\geq 1.20$ & $\geq 2.00$ & $\geq 4.75$ \\
$\Gamma_{\tau, \kappa}=1.5$ & $\gamma^{(\tau, \kappa)}$ & $\geq 5.30$ & $\geq 8.33$ & $\geq 18.9$ \\
$\alpha \cap \beta \cap \gamma$ & $\geq 5.30$ & $\geq 8.33$ & $\geq 18.9$ \\
\hline \hline
\end{tabular}

This table reports the maturity intervals for which the difference between the coefficients of the reverse quadratic regression $R_{t}^{\left(\tau^{*}, \kappa^{*}\right)}=c^{\left(\tau^{*}, \kappa^{*}\right)}+\beta^{\left(\tau^{*}, \kappa^{*}\right)} R_{m t}+\gamma^{\left(\tau^{*}, \kappa^{*}\right)} R_{m t}^{2}+\eta_{t}$ and their respective target values falls below a specific tolerance level. We set $t_{0} l_{\beta}=\theta / \hat{t}_{R_{m}}, \operatorname{tol}_{\gamma}=\theta / \hat{t}_{R_{m}^{2}}$ and $\operatorname{tol}_{\alpha}=$ $\theta\left(\frac{\left|\bar{R}_{m}\right|}{\hat{t}_{R_{m}}}+\frac{\bar{R}_{m}^{2}}{\hat{t}_{R_{m}^{2}}}\right)$ with three possible values of $\theta$. Tested option maturities range between 1 and 52 weeks. The last row represents the intersection between the intervals set for each coefficient. 